\title{
Service Oriented Architecture for Remote Sensing Satellite Telemetry Data Implemented on Cloud Computing
}

\author{
Dr. Abdelfattah E-Sharkawi \\ Al - Azhar University, Cairo, Egypt \\ E-mail:sharkawi_eg@yahoo.com \\ Dr. Ahmed Shouman \\ Menoufia University, Cairo, Egypt \\ E-mail: ahmedshouman13@yahoo.com
}

Eng. Sayed Lasheen

National authority for remote sensing and space science (NARSS), Cairo, Egypt

E-mail: sayedlasheen@yahoo.com

\begin{abstract}
This paper articulates how Service Oriented Architecture (SOA) and cloud computing together can facilitate technology setup in Telemetry (TM) processing with a case study from the Egyptian space program (ESP) and a comparative study with space situational awareness (SSA) program in European space agency (ESA), Moreover, this paper illustrates how cloud computing services and deployment models enable software and hardware decoupling and making flexible TM data analysis possible. The large amount of available computational resources facilitates a shift in approaches to software development, deployment and operations.
\end{abstract}

Index Terms - Cloud Computing, Service Oriented Architecture, Telemetry Data, National Authority for Remote Sensing and Space Science (NARSS)

\section{Introduction}

\subsection{Space system Components}

Any space system can be represented as two components. First is a space segment such as Low Earth Orbit (LEO) satellites [1][2]

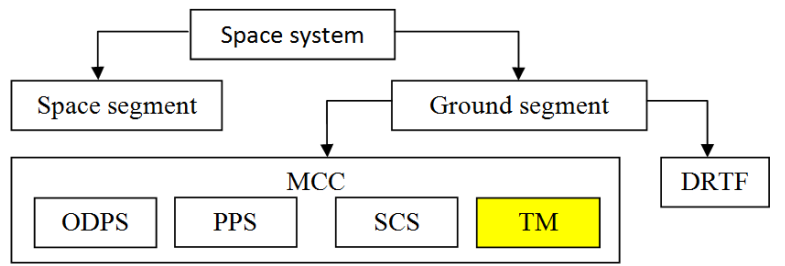

Fig. 1: Space system components
The second is a ground segment which has two parts first is Data Reception and Transmission Facilities (DRTF) that is responsible to communicate with the space segment and second part is Mission control center (MCC) that is responsible to plan payload missions and receive TM data, analy ze them and take in flight control decisions. Figure 1summarizes this structure.

\subsection{Low Earth Orbit (LEO) Remote Sensing Satellites}

Among the important applications of LEO satellites is remote sensing. Satellites are controlled by a Ground Station on earth that sends commands and receives telemetry from satellite to maintain proper operation. LEO satellites could be visible for only a period of time from the point of view of an observer on earth. They can send data to the Ground Station when they pass by a Ground Station zone. Therefore, the Ground station plays a very important role in the communication process with satellites ${ }^{[2]}$

\subsection{Telemetry (TM ) Data}

Data transmitted from a satellite during satellite's ground contact involve not only image data but also housekeeping telemetry data including information about satellite health which comprises a set of satellite status indicators and sensors read outs such as electric current of the on-board equipment and temperature etc. [2] 


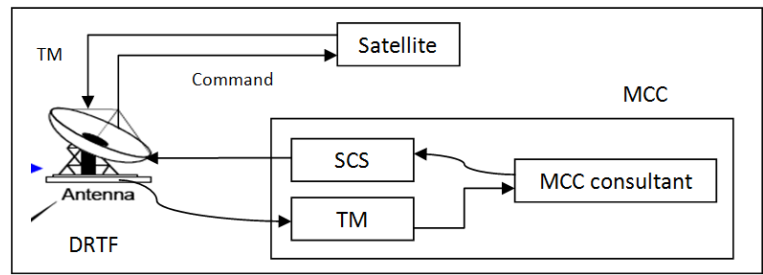

Fig. 2: MCC decision support mechanism

TM subsystem and Satellite Control Subsystem (SCS) together are considered as a decision support system in MCC. If MCC discovers that some mistakes occur through the quick satellite health checking from TM report, MCC normally take the suitable decision by uploading the corresponding commands through SCS. Figure 2 summarizes this decision support mechanism.

\subsection{Service Oriented Architecture (SOA)}

In essence, it is a way of designing a software system to provide services to either end-user applications or other services through published and discoverable interfaces. The basic service oriented architecture (SOA) defines an interaction between software agents as an exchange of messages between service requesters (clients) and service providers. [3] [4] Clients are software agents that request the execution of a service. Providers are software agents that provide the service. Agents can be simultaneously both service clients and providers. Providers are responsible for publishing a description of the service(s) they provide. Clients must be able to find the description(s) of the services they require and must be able to bind to them. The basic SOA shown in Figure 3 does not only represent the architecture for services but also a relationship of three kinds of participants; namely the service provider, the service discovery agency, and the service requestor (client). The interactions involve publish, find and bind operations.

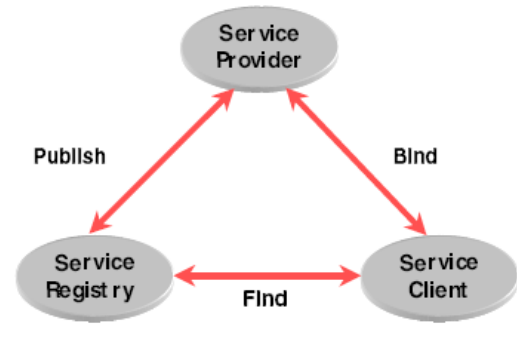

Fig. 3: basic SOA

\subsection{Cloud Computing}

Cloud computing is a model for enabling ubiquitous, convenient, on-demand network access to a shared pool of configurable computing resources (e.g., networks, servers, storage, applications, and services) that can be rapidly provisioned and released with minimal management effort or service provider interaction. This cloud model promotes availability and is composed of five essential characteristics, three service models, and four deployment models

Essential Characteris tics [5] [6] [7] [8] [9] [10]:

- On-demand self-service a consumer can unilaterally provision computing capabilities, such as server time and network storage, as needed automatically without requiring human interaction with each service's provider.

- Broad network access. Capabilities are available over the network and accessed through standard mechanis ms that promote use by heterogeneous thin or thick client platforms (e.g., mobile phones, laptops, and PDAs).

- Resource pooling. The provider's computing resources are pooled to serve multiple consumers using a multi-tenant model, with different physical and virtual resources dynamically assigned and reassigned according to consumer demand. There is a sense of location independence in that the customer generally has no control or knowledge over the exact location of the provided resources but may be able to specify location at a higher level of abstraction (e.g., country, state, or datacenter). Examples of resources include storage, processing, memory, network bandwidth, and virtual machines.

- Rapid elasticity. Capabilities can be rapidly and elastically provisioned, in some cases automatically, to quickly scale out, and rapidly released to quickly scale in. To the consumer, the capabilities available for provisioning often appear to be unlimited and can be purchased in any quantity at any time.

- Measured Service. Cloud systems automatically control and optimize resource use by leveraging a metering capability at some level of abstraction appropriate to the type of service (e.g., storage, processing, bandwidth, and active user accounts). Resource usage can be monitored, controlled, and reported, providing transparency for both the provider and consumer of the utilized service.

\section{Problem Definition}

The first Egyptian remote sensing satellite (Egypt sat-1) was launched to LEO in 2007 by Egyptian Space program (ESP). In order to maintain proper satellite operation, Egyptian Ground control Station was

Established to monitor and control the satellite in its Orbit via DRTF and MCC. Since LEO satellites are visible for only a period of time from the point of view of an observer on earth, they can send TM data to the Ground stations only when they pass by a DRTF zone of theses ground stations as shown in Figure (4-a)

Since ESP got only one ground station in Cairo, Egypt sat-1 could only be seen by DRTF four times per day. As many countries, if ESP decided to build a 
number of DRTF's around the country to cover most of satellite orbits as shown in Figure (4-b). It can monitor other satellites which belong to other partners (countries, small organizations, universities) which has the same satellite series. Moreover, it will use its all hardware and software resources in the ground segment to create a new business model as (pay - per - use) to develop organization assets. According to this proposal, two problems will appear. First is how to handle the TM data for other satellites in the same series? Second is how to design a SOA for TM subsystem to introduce the TM data analysis service and how to implement it on cloud computing?

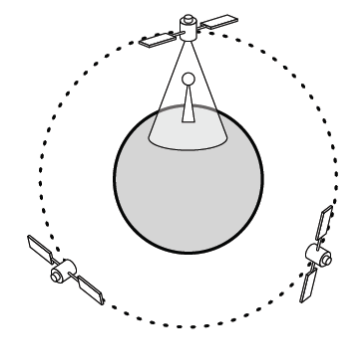

(a) Dedicated station

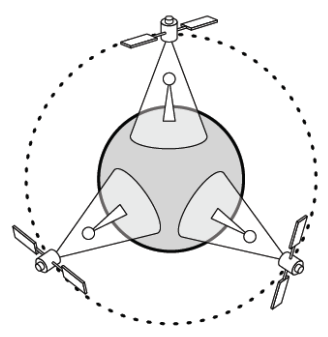

(b) Multiple stations

Fig. 4: DRTF zone

This paper focuses on the second problem which considered as a good case study to implement a huge data on cloud computing

\section{Proposed Solution}

Taking the TM packet structure defined by European cooperation for space standardization $(\mathrm{ECSS})^{[11]}$ into consideration, and using modular object oriented techniques to design TM software this will make standardization in TM data handling problem and offer an application programming interface (API), which enables the software engineers to extend the system and adapt it to their project requirements.

So the following generic steps ${ }^{[12]}$ could be considered to handle TM data

1. Receive the data stream from the satellite

2. Extract the individual TM frames out of the data stream.

3. Extract the raw value of every single TM data from the Frame.

4. Apply the calibration routines to retrieve the engineering value of the TM data.

5. Visualize the values to the clients through some User Interface.

6. Archive the retrieved TM data.

Based on this generic procedure the system architecture shown in Figure 5 has been proposed, which guaranties the highest level of flexibility and extendibility for different Satellite projects.

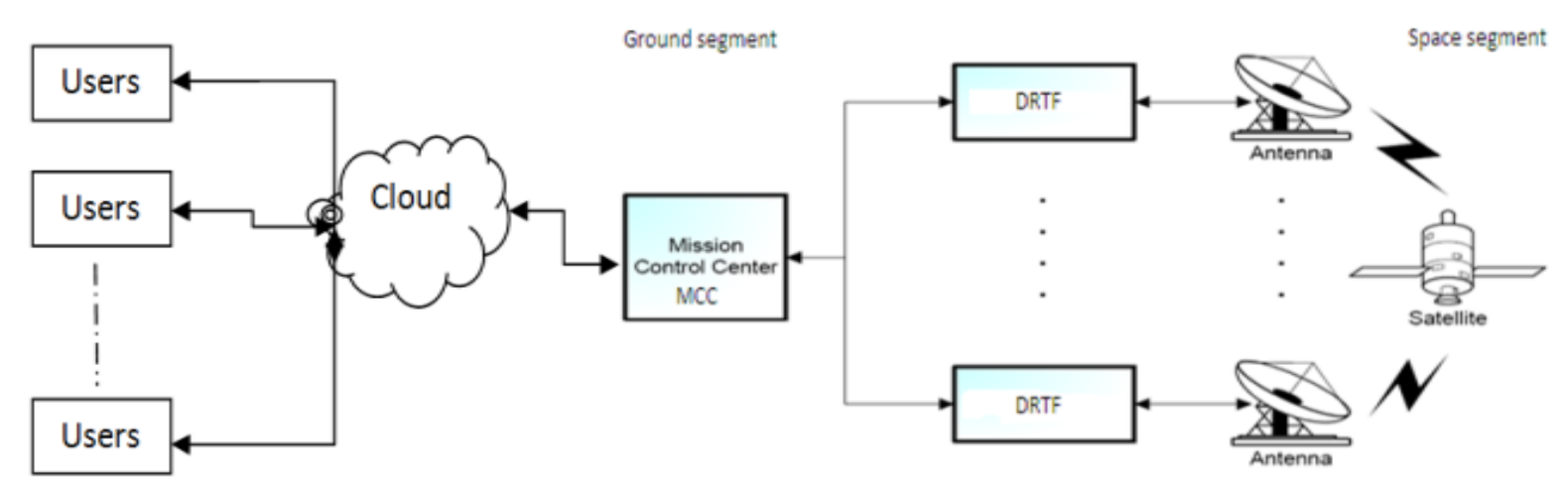

Fig. 5: proposed Architecture of MCC and DRTF

MCC collects all telemetry data (TMD) for each satellite from these DRTF's and makes them available to the partners as a service. Therefore, this research introduces a design of a SOA for TM subsystem to Introduce the TMD analysis service and to study the deployment of these services on cloud computing.

\subsection{System Components}

The system can be divided into the following core software components:
- TM database, which contains the definitions of all TMD. These definitions consist of information about the raw format of the data, i.e. the size in Bytes and Bits, its position in the TM frame, the calibration method to retrieve the engineering value and hard/soft limits for valid values, etc.

- Data handling engine, which establishes a connection to the database and DRTF to carry out the task $\mathrm{Nr} 3,4$ and 6 of the above procedure. Upon a satellite pass it receives telemetry packages sequentially and extracts the telemetry data entities then apply the calibration and archive TMD into database (DB). 
- Reporting facility for monitoring and visualization of the extracted telemetry data to telemetry clients (Task Nr5.).

- Orbit determination package to propagate satellites orbits

\subsection{Data Flow between System Components}

This following paragraph describes the data flow between systemcomponents as shown in Figure 6

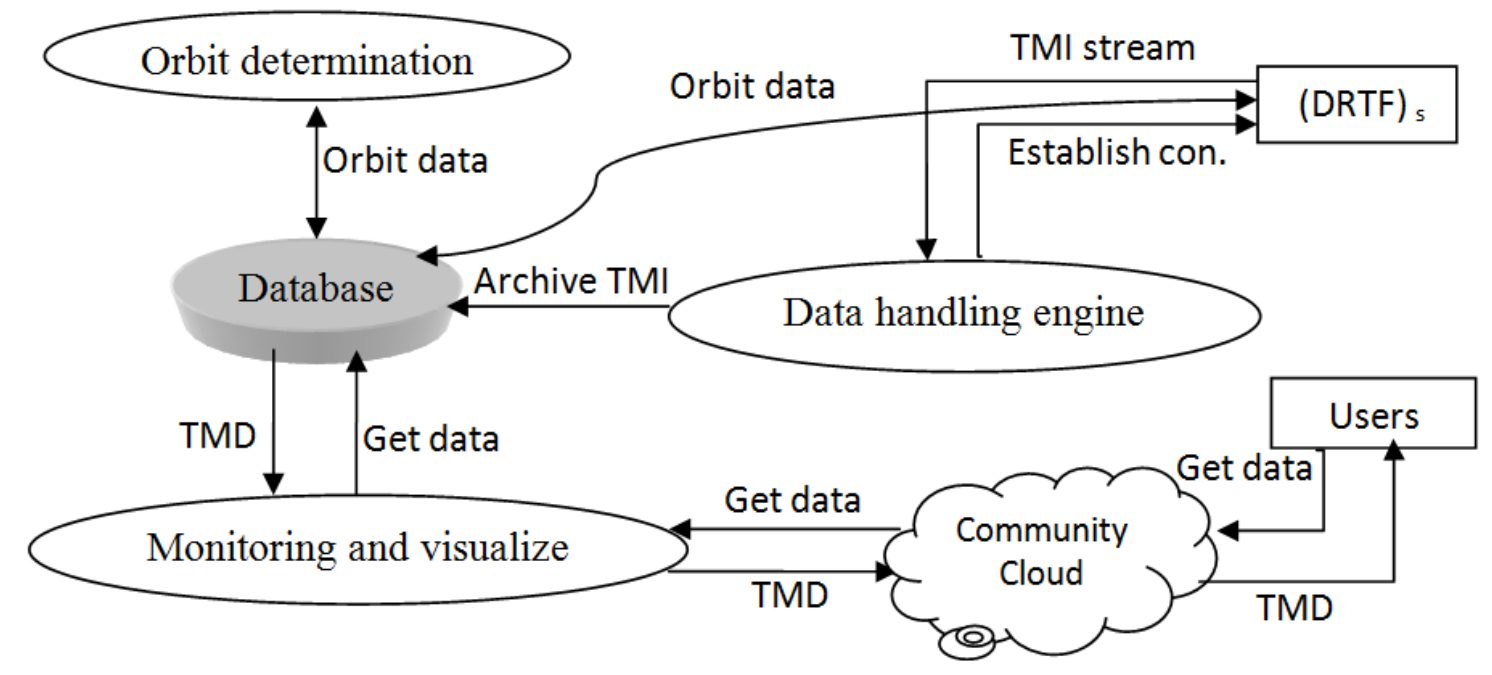

Fig. 6: data flow between TM components

- Orbit determination package propagates the satellite orbits and sends them to MCC database.

- Based on the orbit data, DRTF determines the satellite visibility zones to receive TMD

- Data handling engine establishes the connection with MCC (DB) \& DRTF and extract the received TM packets with parameters' calibration values then archive TMD to database.

\subsection{The Suggested Techniques}

Cloud computing and service oriented technologies will release the opportunity for collaboration and innovation in data utilization which will provide

- High performance for on-demand services

- Fast and easy deployment of services on cloud computing

- Scalability of the system

\section{Service Oriented Architecture (SOA) of TM System}

The design process of a Service-oriented model for TM system are discussed in the following subsections

\subsection{Services Identification Phase Has Two Steps}

\subsubsection{Functional decomposition}

- Determination of Satellite orb its or Calcu late satellite orbits using satellite two line elements then Define the DRTF zone which can track the satellite in a period of time (session) with defining its start and end time to receive TM, This function must be finished by orbit determination subsystem in MCC before satellite communication session start.

After the previous function, Tele metry system can do the following functions to provide all proposed Services:

1. Establish a connection to MCC database and to the (DRTF)

2. Extract TM packet format for each subsystem on the satellite

3. Apply the calibration routines to retrieve the engineering value of the TMD.

4. Archiving TMD on DB during session

5. Authentication management.

\subsubsection{Services decomposition}

1. Display TM parameters in tabular format.

2. Visualize the values of parameters to the clients through some User Interface.

3. TMD analysis per defined period of time with graphs

4. TM reporting per period of time 


\subsection{Services Specification}

From the above discussion, the proposed TM system services can be summarized as Follows:

- packet extraction

- data calibration

- Archiving
- presentation

- analysis

Hence, Users or clients can visualize, monitor, and analysis the extracted telemetry data from reporting facility component through cloud community. The process model of all TM functions and services can be generated using unified modeling language (UML) as shown in Figure 7.

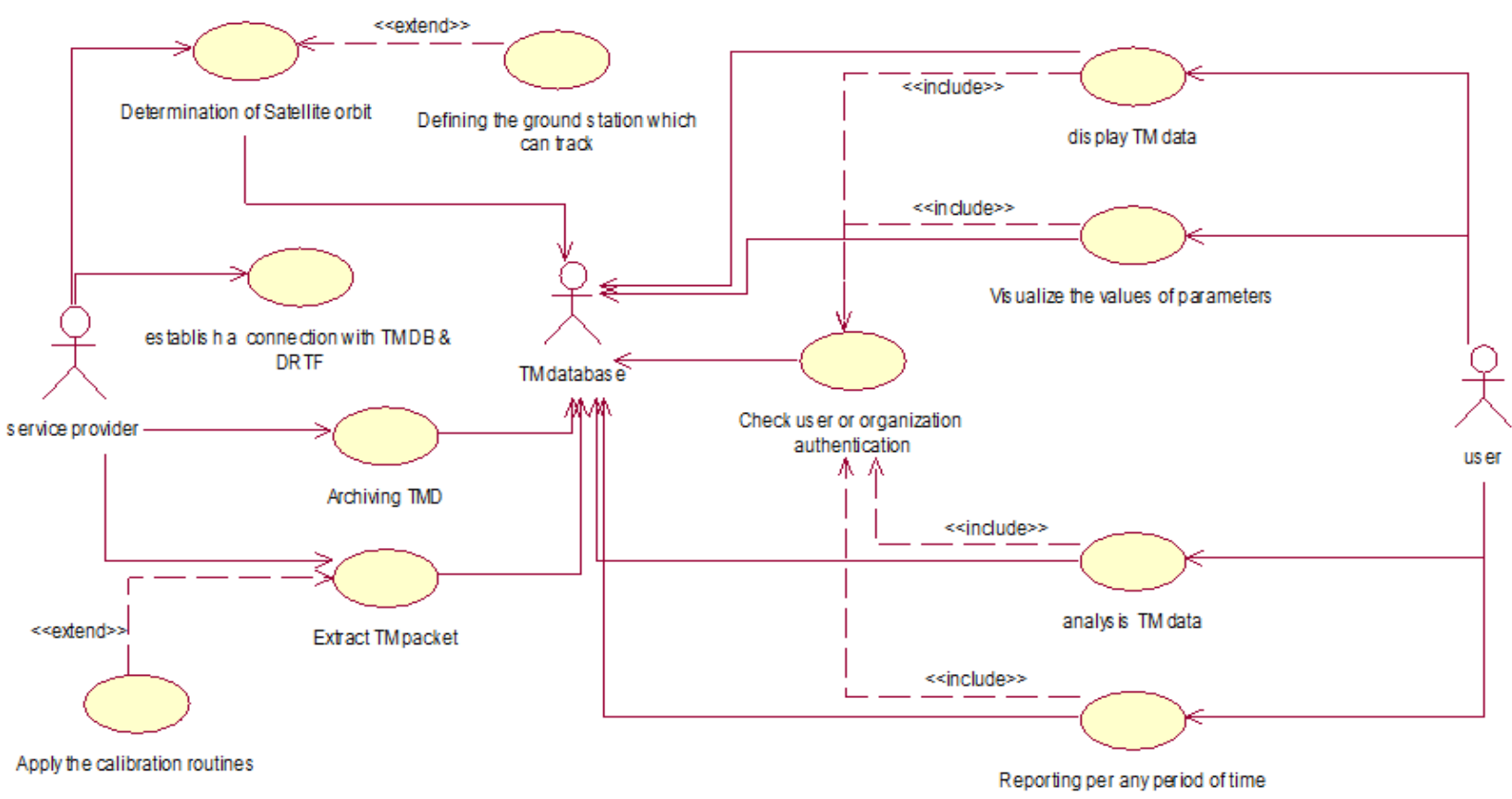

Fig. 7: UML process model for the TM functional and services decomposition

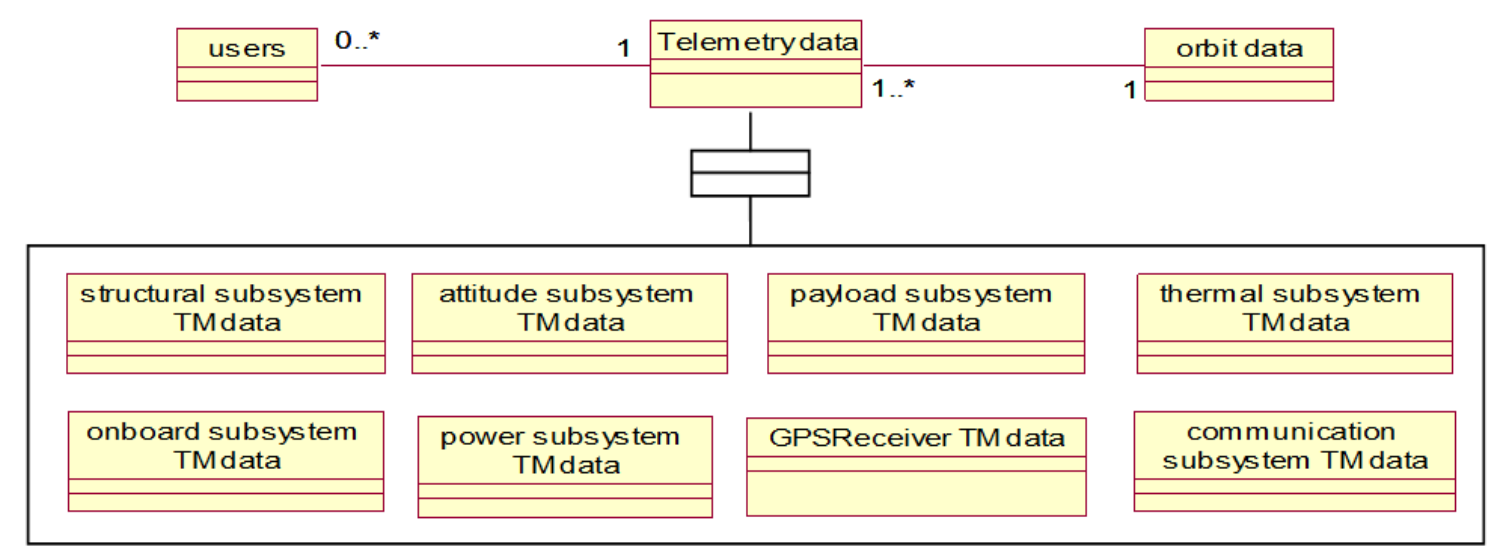

Fig. 8: UML data model for TM system

\subsection{Services Realization}

As explained before there are four software components and this step will recognize the software that realizes a given services, as follows:

\section{- Orbit determination package}

o Define the DRTF which can track the satellite in its zone in a period of time called session with defining its start and end time to receive TM.

\section{- Data handling engine}

o Establish a connection to DB and DRTF

○ Extract TM packet format from TM stream

o Apply the calibration routines to retrieve the engineering value of the TM data.

- Archiving TMD on DB during session to make analysis or statistics 
- Monitoring and visualizing (reporting facility)

o Display TM parameters in table format.

$\circ$ Visualize the values of parameters to the clients through some User Interface.

○ TMD analysis per any period of time with graphs

○ Reporting per any period of time

\section{- TM Database}

This is the main component to realize all previous services.
The UML class diagram shown in Figure 8 shows the data model of the TM database schema.

\subsection{Dynamics of TM Messaging Interaction}

Figure 9 shows the sequence diagram that monitor messaging interaction flow to get a service in two modes; namely real time mode and offline time mode.

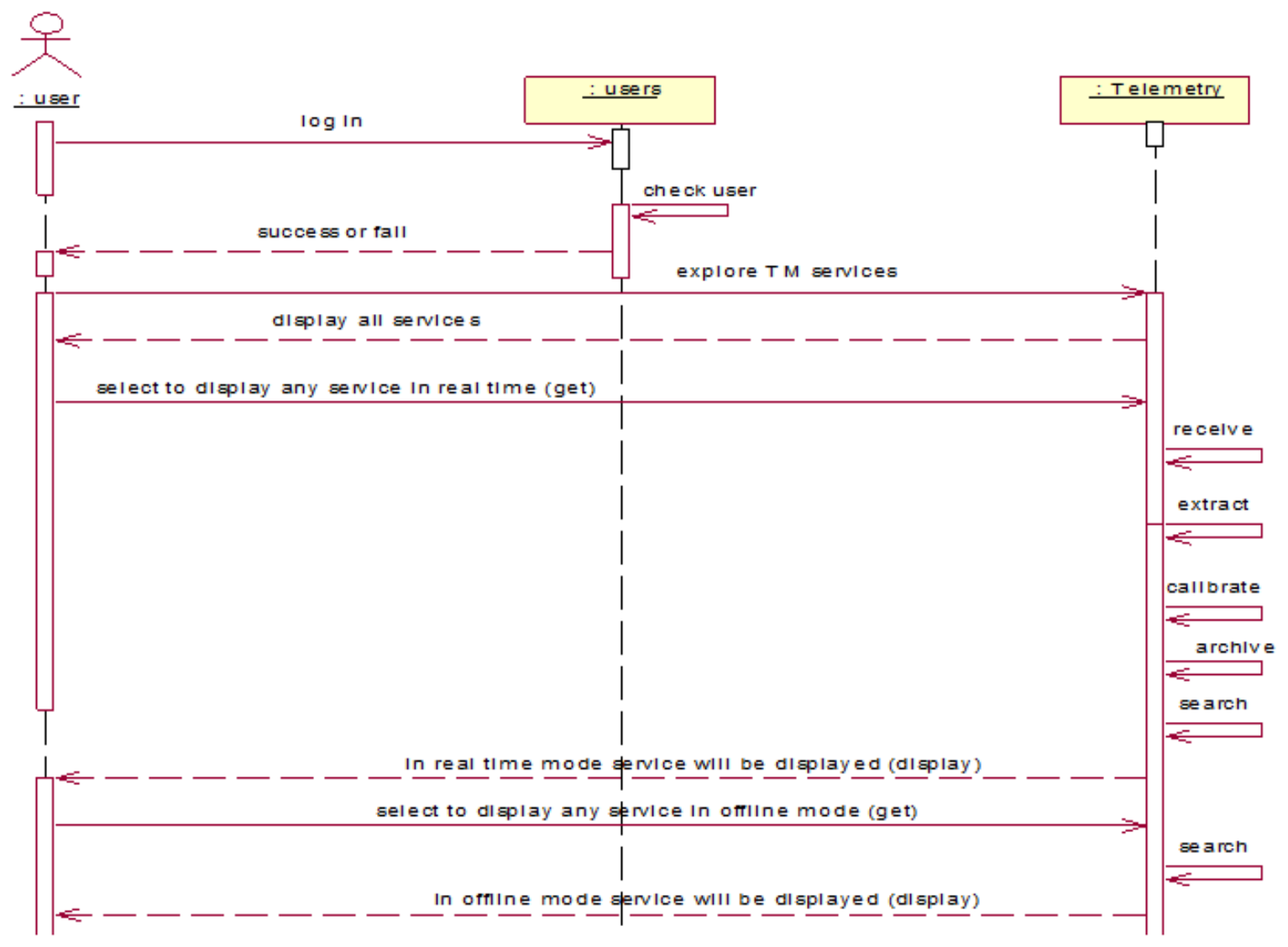

Fig. 9: UML sequence diagram for getting a service

- Sequence of actions of real time mode:

- TMD received from the satellite

○ TMD extracted in data handling server

- Calibrate TMD to its engineering values to be displayed

o Archive TMD into DB

- Search in DB about the user data request to display

\section{- Sequence of actions of offline mode}

o Search in DB about the userdata request

○ Display telemetry data

\subsection{Proposed Service Oriented Architecture (SOA)}

The proposed architecture has three main blocks shown in Figure 10 as follows:

- TM data processing which will establish the connection with (DRTF) during satellite communication session to extract TMD from TM packets with calibration of parameters values and archive it into DB

- Data center or DB has all TM data for all satellites which was archived; users can get any TMD through data administration module. A knowledge base will be used to generate statistics and reports.

- Services provision center this block has users interface to access any service after authentication. 


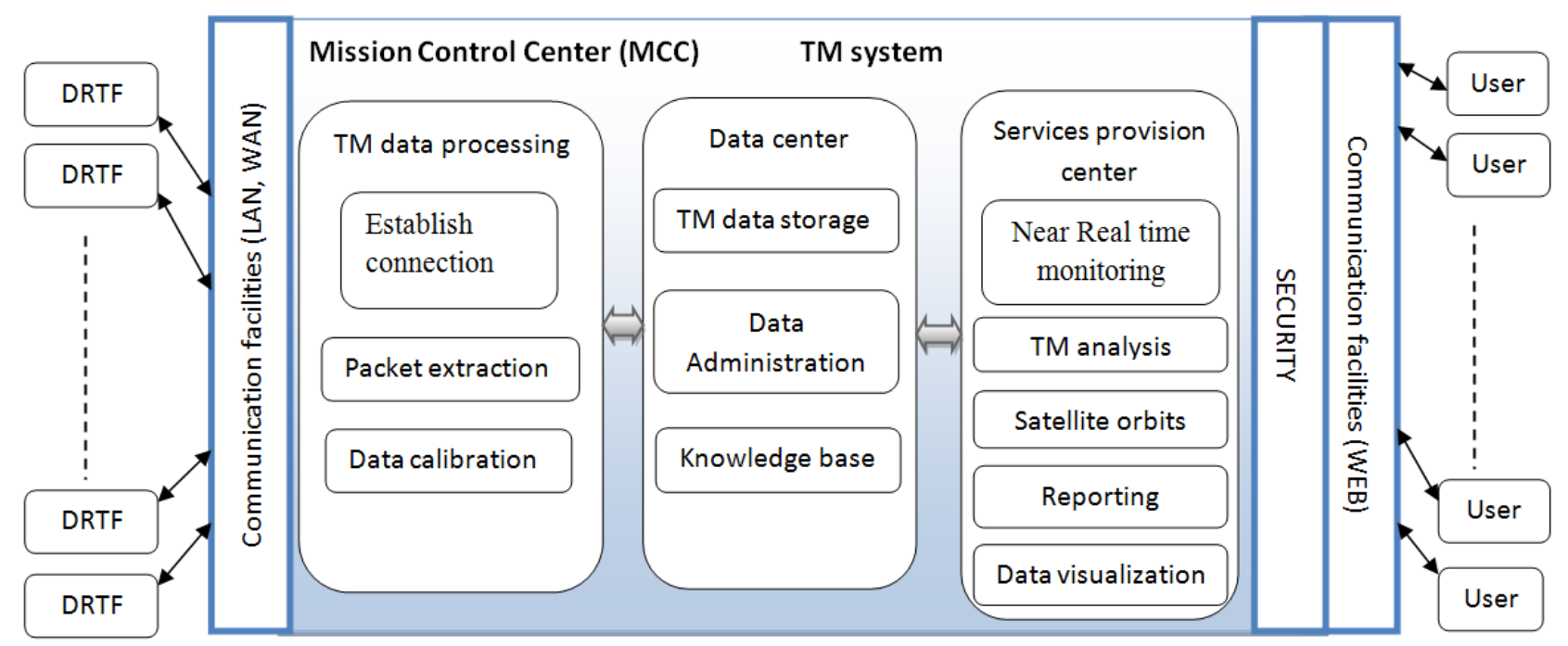

Fig. 10: proposed TM (SOA)

\section{Proposed Cloud Computing Implementation}

\subsection{Cloud Framework}

The First step to implement TM (SOA) on the cloud computing is to define the cloud frame work as shown in table 1

Table 1: cloud frame work

\begin{tabular}{||c||c|c|c||}
\hline $\begin{array}{c}\text { Deployment } \\
\text { model }\end{array}$ & Community Cloud \\
\hline \hline $\begin{array}{c}\text { Essential } \\
\text { characteristics }\end{array}$ & Data as a Service (DaaS) & Software as a Service & Infrastructure as a Service \\
\hline \hline Broad Network Access & Resource Pooling \\
\hline
\end{tabular}

This table shows a community cloud as a deployment model that has three service models:

- Data as a service (Daas), will introduce TM data analys is and visualization.

- Software as a service (Saas), will introduce a TM software tools to the users.

- Infrastructure as a service (Iaas), all ground station resources will be used to introduce all services to the users.

\subsection{Cloud computing Architecture}

The proposed definition of TM cloud computing architecture shown in Figure 11 is divided into four layers to provide differentiating capabilities to the TM systemusers as follows:

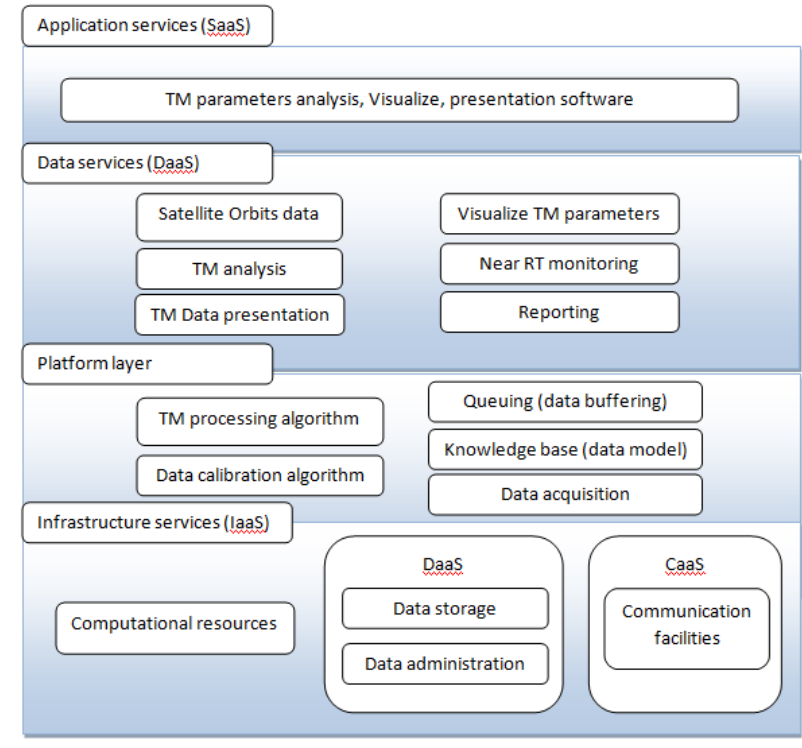

Fig. 11: TM cloud architecture 
- Application services layer

Provide a ready to use software applications, in a SaaS model, such as TMD parameters analysis, TMD Visualization, TMD graph presentation software.

The users can access the services provided by this layer through web-portals.

- Data services layer

Provide all satellite TM data to authenticated users called (Daas).

- Platform services layer

Provide all technical and business algorithms to make data calculation, calibration and processing.

- Infrastructure services layer

Deliver computational resources, communication facilities in communication as a service (Caas) model and data storage for the layers above - called IaaS.

\section{Cloud Computing Simulation}

To provide TM services through cloud computing; Figure 12 shows the needed tools.

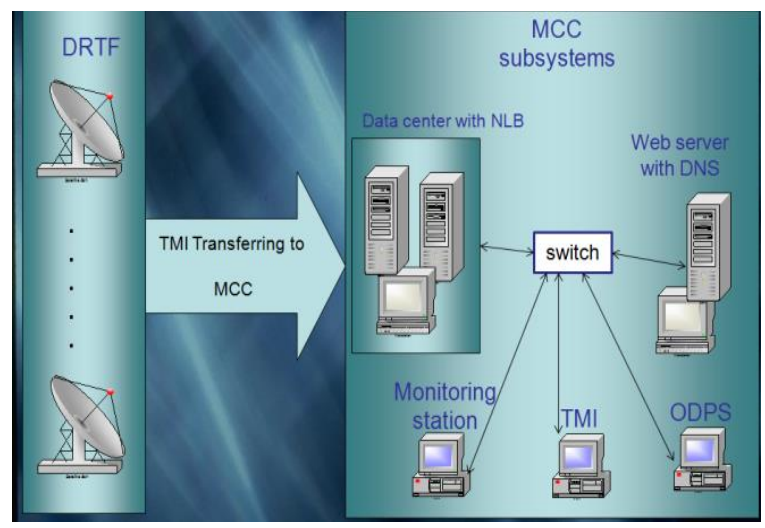

Fig. 12: TM cloud computing simulation components

- Data center

- Satellite data trans mission and receiving facilities (DRTF)

- Networking

- Platform (operating system OS)

- TM data extractor software

- Orbit determination and propagation subsystem (ODPS)

- Web server with domain name system (DNS) to manage Clients services.

- Monitoring workstation to measure quality of services (QOS)

\section{- Data center}

This component was simulated using two database servers working with clustering technique to archive and manage all TM data as shown in Figure 13.

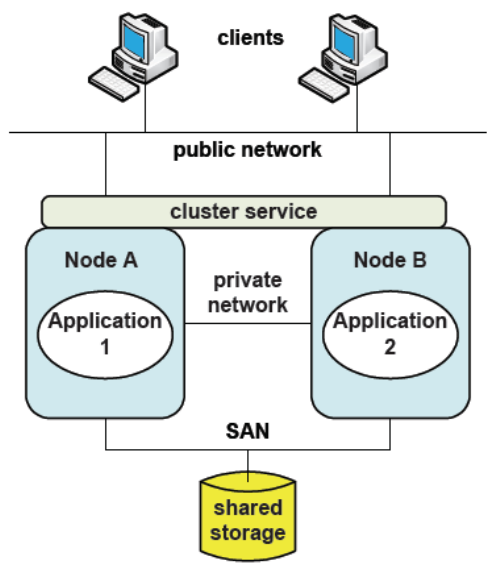

Fig. 13: clustering technique

The group of nodes of each cluster is managed as a single system and shares a common namespace.

\section{- Monitoring workstation}

There are many Cloud Computing Monitoring tools as PRTG tool ${ }^{[13]}$; which has many sensors that can measure and visualize the performance of all resource parameters such as CPU load, memory usage, hard disk, SQL, HTTP etc. as shown in Figure 14

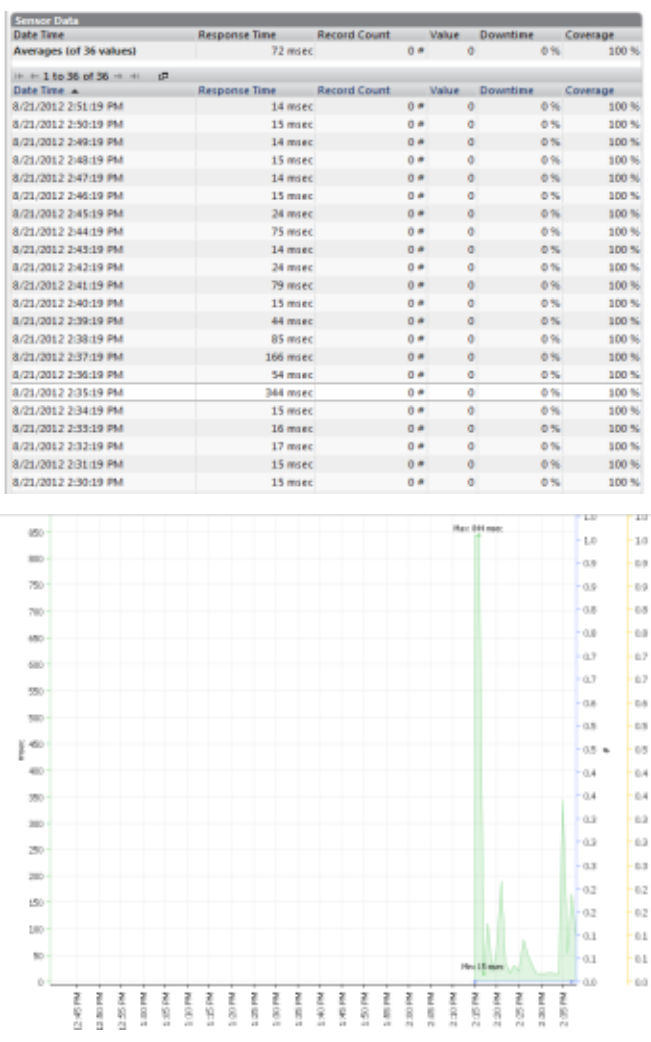

Fig. 14: PRTG network monitoring tool 


\subsection{Cloud Computing Network Performance Parameters Simulation}

The main factors which effect network performance parameters are:

- Internetworking devices

- Type of data being transferred

- Network topology

- Number of users on the network

- User computer

- Server computer

\subsubsection{Network protocol}

OPNET network simulation tool was used as shown in Figure 15 to insure that transmission Control protocol (TCP) is A Reliable, Connection-Oriented, and Byte-Stream Service

This test for FTP service on server side using IPcloud32 node model as IP cloud supporting up to32 serial line interfaces at a selectable data rate through which IP traffic can be modeled. IP packets arriving on any cloud interface are routed to the appropriate output interface based on their destination IP address Choosing Congestion Window Size (bytes) and Sent Segment Sequence Number Statistics, to compare the performance of the algorithms through the analysis of the simulation results

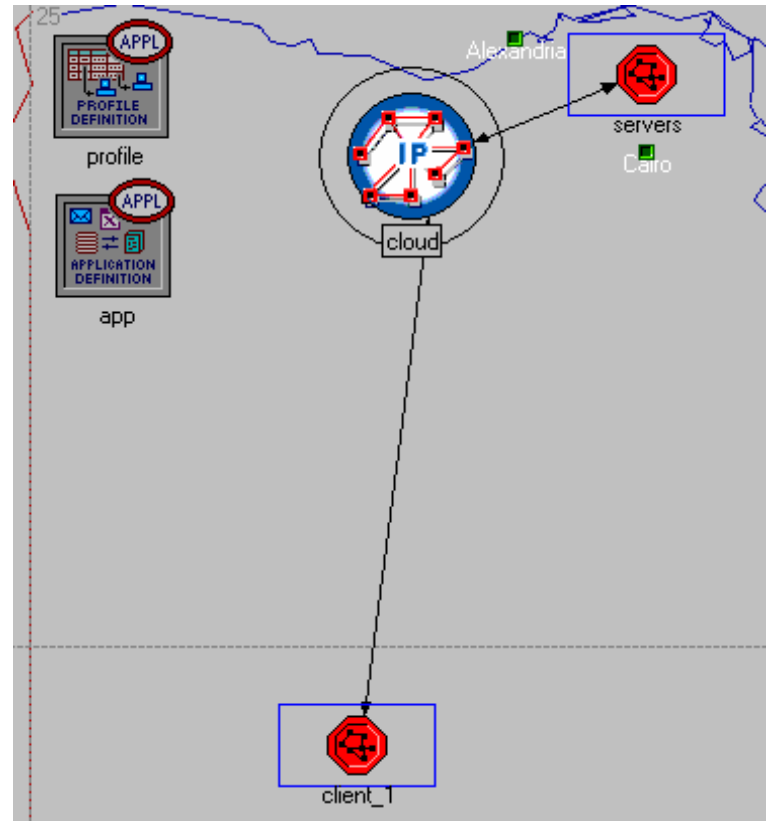

Fig. 15: TCP Simulation

N.B The sent Segment Sequence Number is almost flat with every drop in the congestion window as shown in Figure 16. This insures that TCP guarantees the reliable, in-order delivery of a stream of bytes. It includes a flow-control mechanis $m$ for the byte streams that allows the receiver to limit how much data the sender can transmit at a given time. In addition, TCP implements a highly tuned congestion-control mechanism.

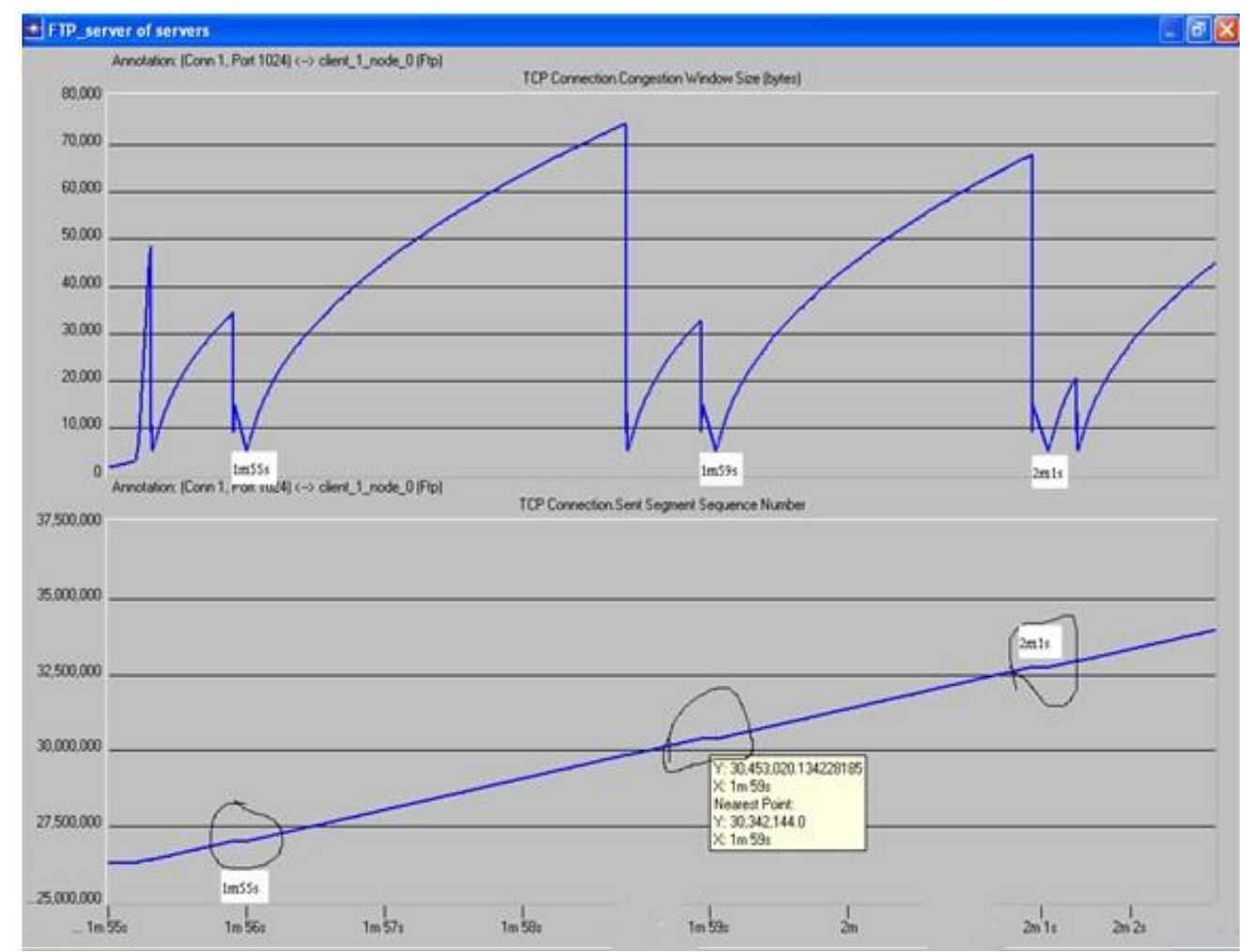

Fig. 16: TCP sent segment sequence number compare with congestion window size 


\subsubsection{Network Throughput}

Throughput refers to actual measured bandwidth, at a specific time of day, using specific Internet routes, and while a specific set of data is transmitted on the network. ${ }^{[14][15]}$

Bandwidth is the measure of the amount of information that can move through the network in a given period of time. Therefore, the amount of available bandwidth is a critical part of the specification of the network. A typical LAN might be built to provide $100 \mathrm{Mbps}$ to every desktop workstation, but this does not mean that each user is actually able to move 100 megabits of data through the network for every second of use. This would be true only under the most ideal circumstances.

\section{Maximum TCP throughput $=$ TWS $/$ RTT bps}

Where

TWS $=$ TCP window size or receiver buffer size

RTT $=$ round-trip time.

bps $=$ bits per second

\section{Data transfer time calculation,}

Theoretical $\mathbf{T}=\mathrm{S} / \mathrm{BW}$ sec $\& \&$ Actual $\mathrm{T}=\mathrm{S} / \mathrm{P}$ sec

Where

$$
\begin{aligned}
& \mathrm{T}=\text { data Transfer time } \\
& \mathrm{S}=\text { size of file } \\
& \mathrm{BW}=\text { theoretical bandwidth } \\
& \mathrm{P}=\text { actual bandwidth at the moment of transfer } \\
& \mathrm{Sec}=\text { second }
\end{aligned}
$$

End-to-end delay refers to the time taken for a packet to be transmitted across a network from source to destination.

$$
D_{\text {end-end }}=\mathbf{N}\left[d_{\text {Trans }}+d_{\text {prop }}+d_{\text {proc }}\right]
$$

Where

$$
\begin{aligned}
& D_{\text {end-end }}=\text { end-to-end delay } \\
& d_{\text {Trans }}=\text { transmis sion delay } \\
& d_{\text {prop }}=\text { propagation delay } \\
& d_{\text {proc }}=\text { proces sing delay }
\end{aligned}
$$

$\mathrm{N}=$ number of links (Number of routers +1 )

Note: neglected queuing delays.

Each router will have its own $\mathrm{d}_{\text {Trans }}, \mathrm{d}_{\text {prop }}$, and $\mathrm{d}_{\text {proc }}$

Assume that no TCP window scaling option found, so TCP window size will be used as 64 Kbytes. Then,
Round Trip Time (RTT) is the main factor to decide TCP throughput between locations.

Table 2: RTT and throughput relation

\begin{tabular}{|c|c|c|}
\hline $\begin{array}{c}\text { RTT } \\
\mathbf{m s}\end{array}$ & $\begin{array}{c}\text { TCP throughput } \\
\text { bps }\end{array}$ & $\begin{array}{c}\text { TCP throughput } \\
\text { Mbps }\end{array}$ \\
\hline 10 & 52428000 & 52 \\
\hline 20 & 26214000 & 26 \\
\hline 50 & 10485600 & 10 \\
\hline 100 & 5242800 & 5.2 \\
\hline 150 & 3495200 & 4.3 \\
\hline 200 & 2621400 & 2.5 \\
\hline 300 & 1747600 & 1.7 \\
\hline 500 & 1048560 & 1 \\
\hline
\end{tabular}

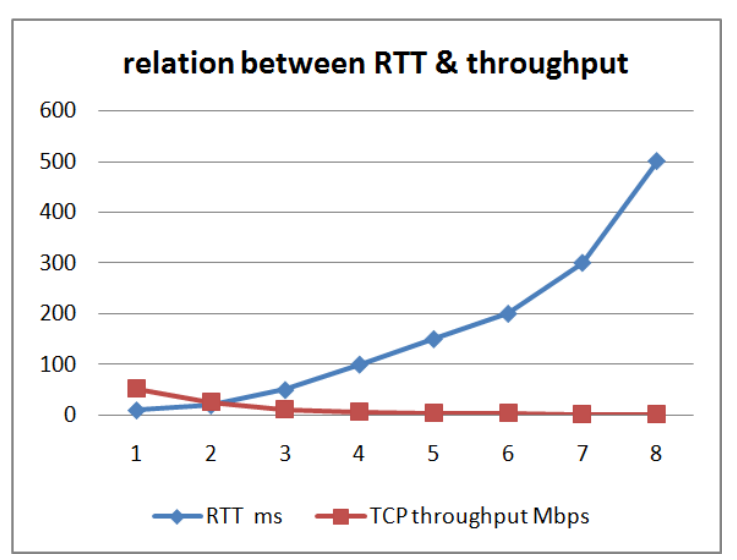

As it clear from table 2 and graph, with the greater RTT throughput is lower.

\subsubsection{Network Utilization}

Network utilization is the ratio of current network traffic to the maximum traffic that the port can handle. It indicates the bandwidth use in the network. While high network utilization indicates the network is busy, low network utilization indicates the network is idle.

\subsubsection{Response time}

Refers to the time taken to execute a job it depends on resource characteristics and network utilization this will clear after simulation for FTP down load response time, web response time and database query response time. Networks of different types or in different topology have different theoretical peek value under general conditions.

\subsection{Cloud Computing Services Performance} Parameters Simulation 
Using OPNET network simulation tool the following Cloud services performance parameters was measured

- FTP service which contain TM data and files transfer.

- HTTP or web service
- Database service

- Servers Load

Table 3 contains simulation data which will be illustrated in Figure 17

Table 3: simulation data

\begin{tabular}{|l|l|}
\hline \multicolumn{1}{|c|}{ Simulation parameter } & \multicolumn{1}{c|}{ Value } \\
\hline Protocol & TCP \\
\hline Number of subnets & $(4)$ - one for servers and three for clients \\
\hline Internal subnets links & 100 base T \\
\hline External links & PPP-DS3 \\
\hline Cloud & IP_cloud32 \\
\hline Number of servers & One for data center and the other for HTTP \& FTP each one contains single processor \\
\hline Number of applications & Three - (FTP,HTTP and database ) each client can access all applications in serial order \\
\hline HTTP application & Heavy browsing \\
\hline Database application & High access \\
\hline
\end{tabular}

\begin{tabular}{|c|c|}
\hline ث $(\mathrm{Ftp})$ Table & $=0 \times$ \\
\hline Altribute & Value \\
\hline Command Mix (Get/Total) & $100 \%$ \\
\hline Inter-Request Time (seconds) & constant $(3600)$ \\
\hline File Size (bytes) & constant $(10000000)$ \\
\hline Symbolic Server Name & FTP Servet \\
\hline Type of Service & Best Effort (0) \\
\hline
\end{tabular}

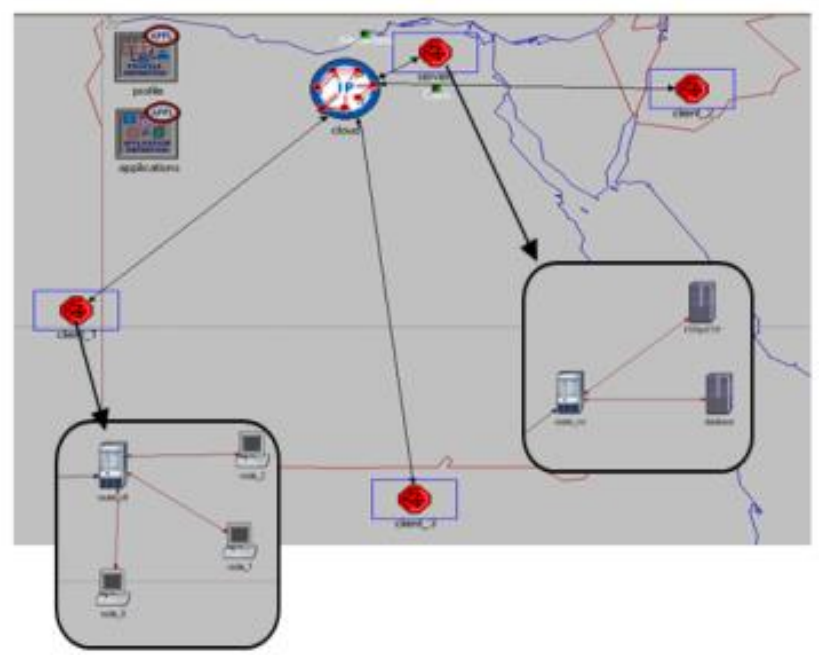

Fig. 17: main scenario configurations and FTP application parameters table

\subsubsection{The relation between link background utilization and other performance parameters}

By modifying PPP_DS3 link background utilization between server's subnet and cloud in scenario (2) as shown in Figure18

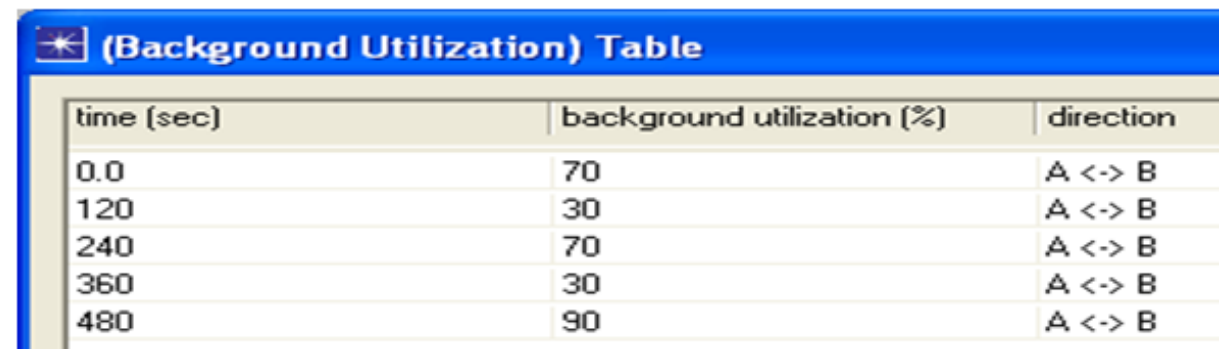

Fig. 18: Background utilization

Throughput of the DB server load will be changing according to utilization as HTTP server performance load will increase as shown in Figure 19 


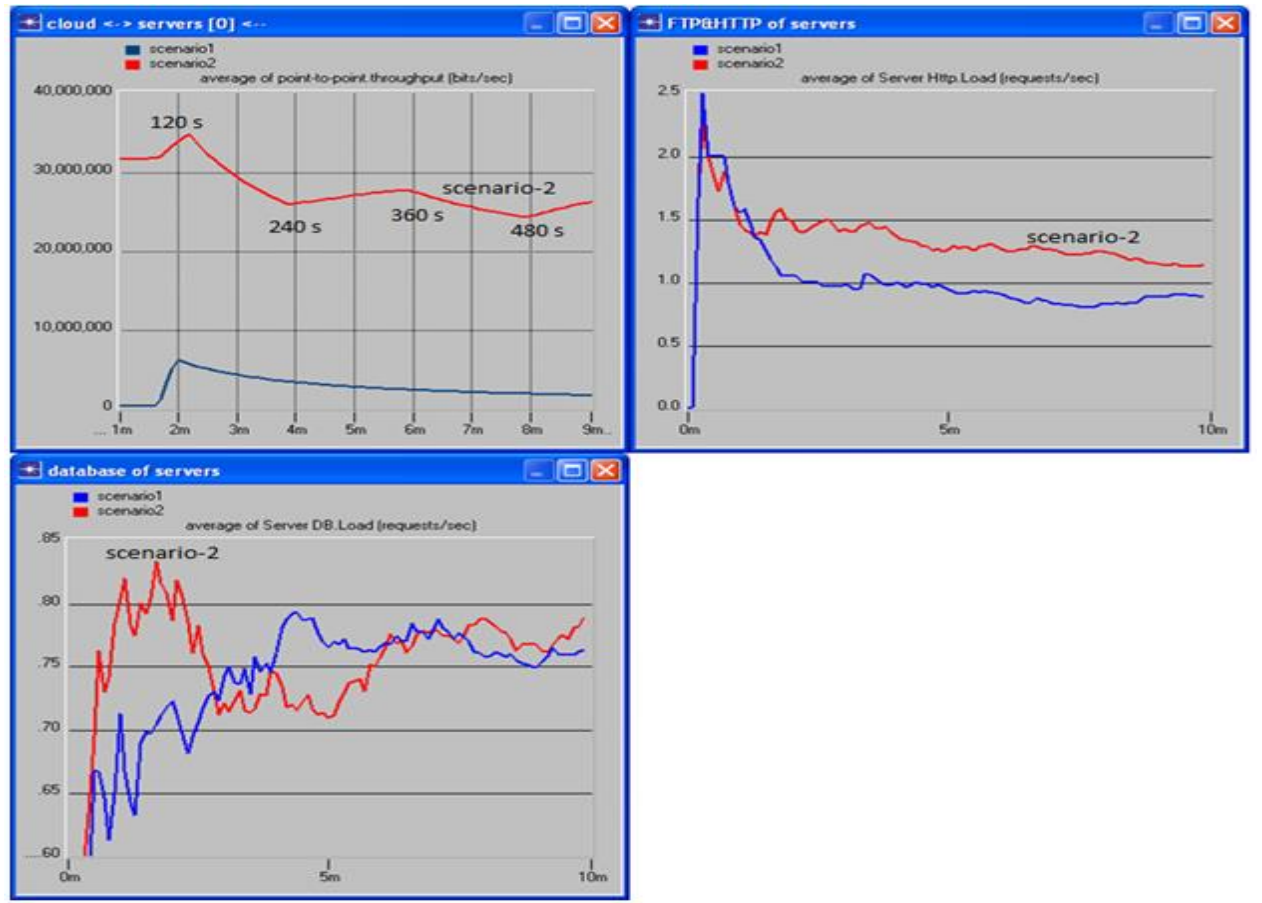

Fig. 19: results comparing

\subsubsection{The relation between number of clients and other performance parameters}

By increasing the number of clients in each subnet from three clients in scenario (1) to six clients in each subnet in scenario (3), each client can access all applications (FTP, HTTP and database) in serial operation mode.

Database server and FTP\&HTTP server load will increase as shown in Figure 20

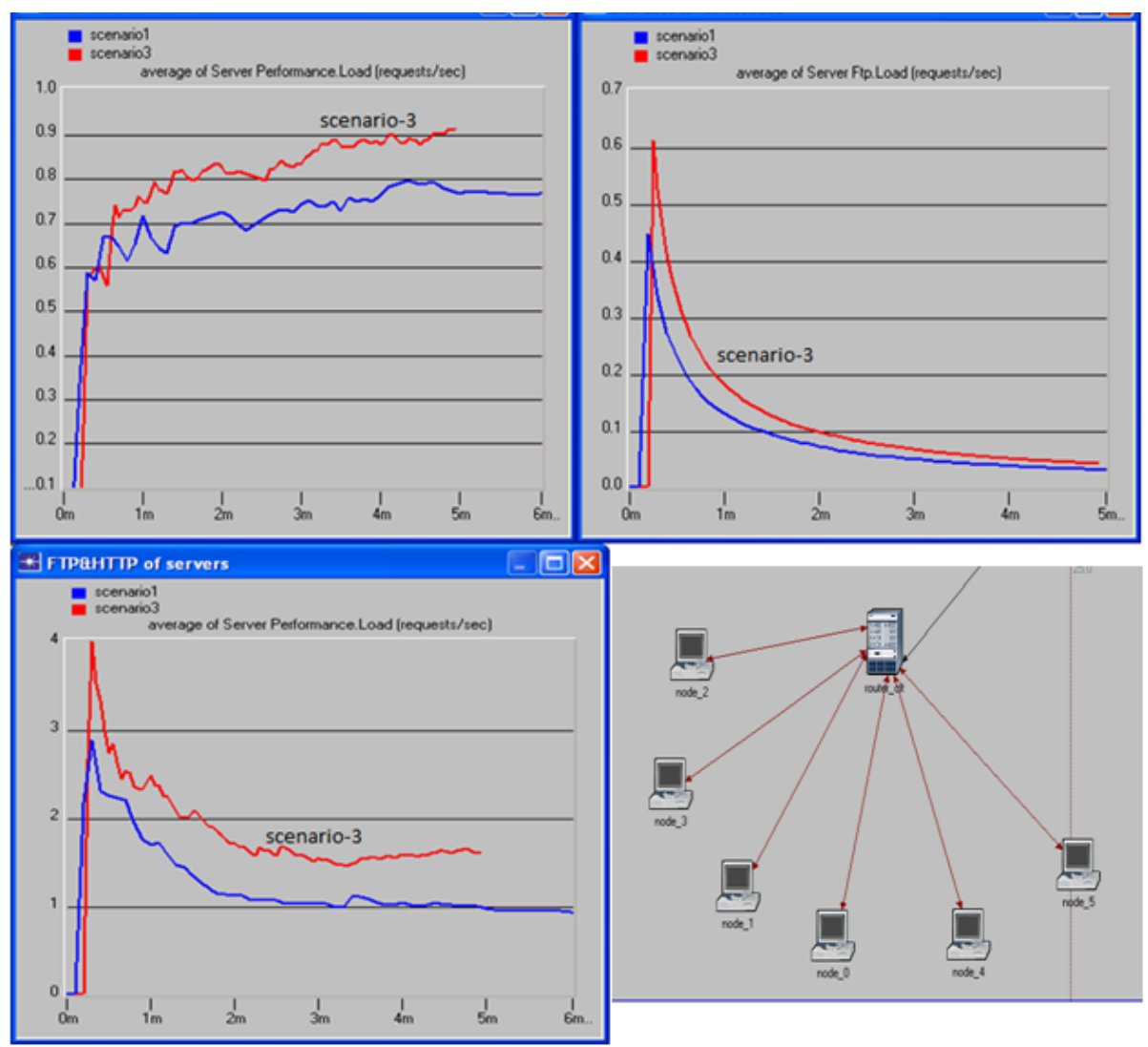

Fig. 20: results comparing 


\subsubsection{The relation between servers enhancement and other performance parameters}

Enhancing database server and (HTTP\& FTP) server performance by increasing the number of processors to three processors in scenario (4) instead of single processor in scenario (3)

Task processing time in database, FTP and HTTP servers will decreased as shown in Figure 21

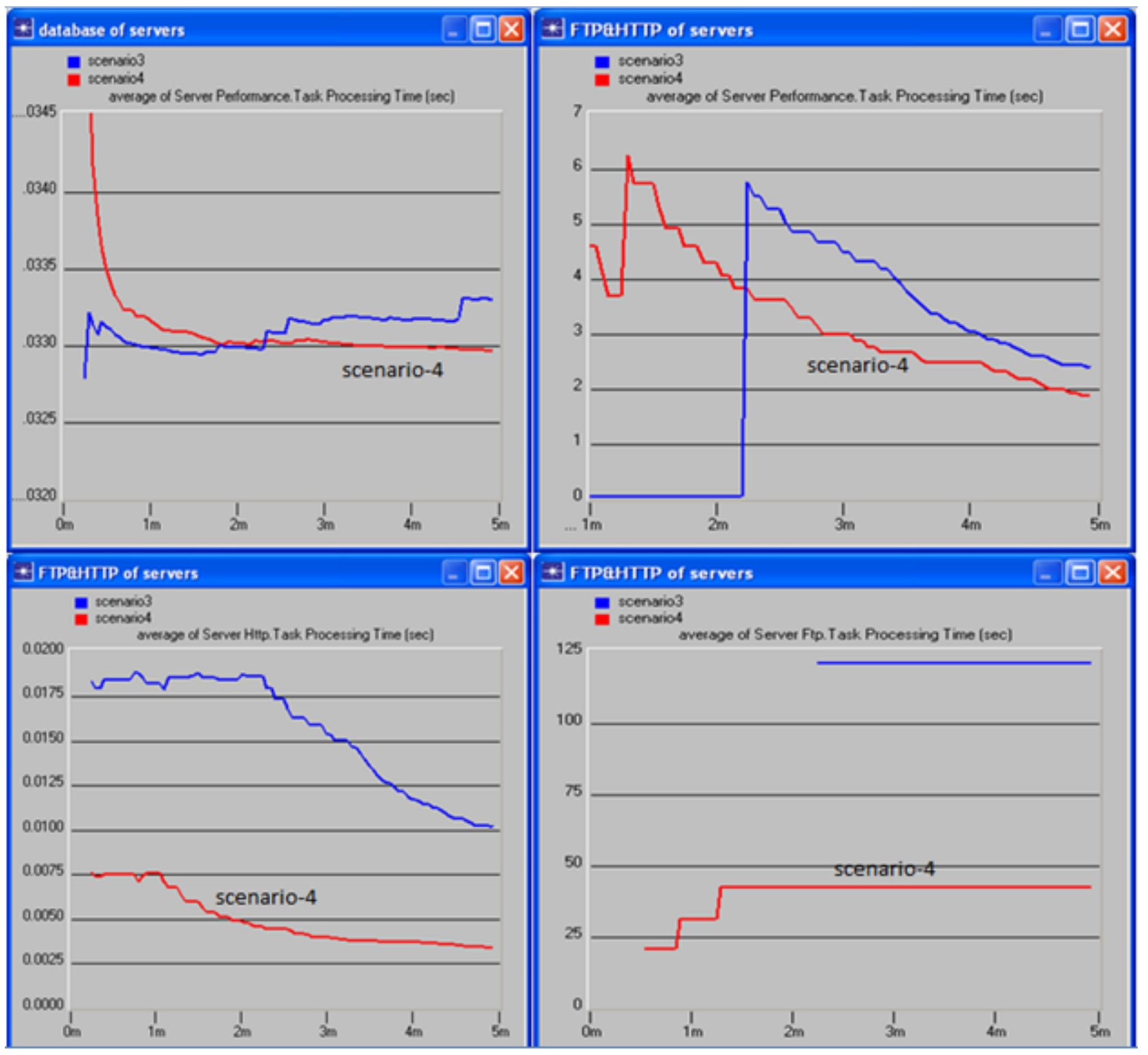

Fig. 21: results comparing

\section{Comparative Study}

The Eu ropean Space Agency (ESA) has launched the Space Situational Awareness (SSA) program, an initiative combining three different segments focusing on near earth objects (NEO), space surveillance \& tracking of man made objects (SST) and space weather (SWE). SSA faces a challenging computational and data management landscape, given the volume of data and processing power required to fulfill its missions. ${ }^{[16]}$ Using service oriented and cloud computing ; T.Michelbach and ET.Al. Introduced an approach to SSA manipulate its resources on investments and initiatives related to its missions.
In the following paragraph, we are going to present a comparative study of our cloud model presented in this paper to that of SSA.

Our model articulates a service oriented architecture of remote sensing satellites TM data (as a Case study) with its cloud computing architecture to introduce new business model to Egyptian Space program as well as other applications dealing with huge amount of data to be introduced as A service to its partners, Compared to SSA, our model introduces a difference concerning the following measures: 
Maintainability - $\quad$ Our model increased maintainability by definition of standardized requirements

Interoperability - Our model increased interoperability by standardized service and data models

SOA - Our model has software components used in processing with Data flow between software components (SSA does not)

Cloud architecture - Our model used Data as a service layer (SSA used Platform as a service).

Our model which offers computation to only in one layer (rather than in all layers as in SSA model)

\section{Conclusion}

Implementing the proposed service oriented architecture (SOA) of satellite tele metry data (TMD) on cloud computing was a very good case study in this research to illustrate that Cloud computing and SOA techniques have the ability to deploy large amount of data as a service to the clients with substantial scalability and elasticity. We introduced a mathematical analysis procedure to show how the performance of cloud would be affected by the Quality of services (QOS) of the network.

Simulation results were collected using OPNET for studying the performance of the cloud based on the network protocol as TCP; the effect of RTT on network throughput, Network utilization and response time. On the application level, simu lation results that affect cloud performance were also collected such as link background utilization, the number of clients and the number of processors. To show the added values of this research, a comparative study with a similar work done by the European space agency (Space Situational Awareness) was also introduced. Therefore, The organizations working in fields such as space, petroleum, multimedia... etc. which offers a huge amount of data (to be introduced as a service to its partners) can use the illustrated procedures in this research to implement cloud computing facilities with high (QOS).

\section{References}

[1] http://www.fas.org/spp/military/docops/army/ref_t ext/chap07a.htm

[2] $\mathrm{Yu} \mathrm{Du}$, "A Satellite Ground Station Control System", Technical University of Denmark , Informatics and Mathematical Modeling, Lyngby 21 October 2005 IMM-THESIS-2005-86, pp. $17,18,26,27,34$

[3] Mike P. Papazoglou, " Service -Oriented Computing: Concepts, Characteristics and
Directions", Proceedings of the Fourth International Conference on Web Information Systems Engineering (WISE'03), PP. 3 , VOL 0 7695-1999-7/03 \$17.00, 2003 IEEE

[4] AsadullahShaikh, MunibaMemon NasrullahMemon ,Muhammad Misbahuddin ," The Role of Service Oriented Architecture in Telemedicine Healthcare System”, IEEE 2009

[5] Peter Mell ,Timothy Grance, “ The NIST Definition of Cloud Computing (Draft) “, NIST , PP. 6,7 , Special Publication 800-145 January 2011

[6] Yuri Demchenko , Cees de Laat, " Defining Generic Architecture for Cloud Infrastructure as a Service Model", The International Symposium on Grids and Clouds and the Open Grid Forum Academia Sinica, Taipei, Taiwan, March 19 - 25, 2011

[7] Joe Weinman, "Mathematical Proof of the Inevitability of Cloud Computing" ,http://www.JoeW ein man.com/Resou rces/Joe_Weinman_Inevitability_Of_Cloud.pdf, 2011

[8] Anthony T. Velte ,Toby J. Velte ,Robert Elsenpeter,“ Cloud Computing A Practical Approach”, ISBN: 978-0-07-162695-8, 2010

[9] Minqi Zhou, Rong Zhang, DadanZeng, WeiningQian, "Services in the Cloud Computing Era: A Survey “, IEEE 2010

[10] Michael Behrendt ,BernardGlasner ,Petra Kopp ,Robert Dieckmann ,GerdBreiter ,Stefan pappe ,Heather Kreger, Ali Arsanjani, "Introduction and Architecture Overview IBM Cloud Computing Reference Architecture 2.0 ", IBM , 2011

[11] European cooperation for space standardization (ECSS) "Ground systems and operations Telemetry and telecommand packet utilization " , ECSS - E- 70-41A , 30 January 2003

[12] MehranSarkarati, Keplerlaan, Noordwijk ZH, "A WEB-BASED MODULAR AND FLEXIBLE DATA ACQUISITION AND TELEMETRY MONITORING SYSTEMFOR MICRO SATELLITES “

[13] Paessler AG, "PRTG Network Monitor 9 Getting Started", September 2011

[14] http://basicitnetworking.blogspot.com/2009/11/tho ughput-data-transfer-calculation.html

[15] Andrew Kaczorek, Jason Stowe, “ Maximizing Data Throughput”, ,http://cloudcomputing.sys con.com/node/1590356/print ,10/3/2012

[16] T. Michelbach, B. E. Amstutz, T. T. Usrey, G. Frith , M. Ziegler , C. Tueffers , J. Stogdill, A. ÁguedaMaté ,"Cloud Computing and Service 
Orientation in the SSA programme Space Surveillance services and data in the Cloud" ,European Space Surveillance Conference 2011

\section{Authors' Profiles}

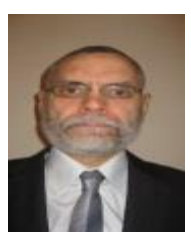

Dr. Abdelfattah E-Sharkawi: Associate professor, Software Engineering ,Al -Azhar University, Egypt; Project Director for the Development of software of Mission Control Center at Egyptian National Agency for Remote Sensing and Space Sciences (NARSS).

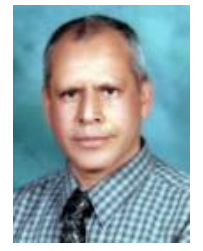

Dr. Ahmed Shouman: Assistant Professor, Department of Engineering and Computer Science, Faculty of Electronic Engineering, Monoufia University, Egypt.

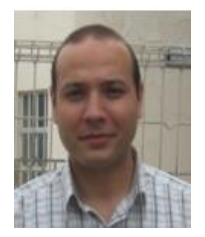

Eng. Sayed lasheen: Telemetry senior engineer, in Egyptian ground control station, Graduate student for master degree for computer science in Menoufia University, major in computer science

How to cite this paper: Abdelfattah El-Sharkawi, Ahmed Shouman, Sayed Lasheen," Service Oriented Architecture for Remote Sensing Satellite Telemetry Data Implemented on Cloud Computing", International Journal of Information Technology and Computer Science(IJITCS), vol.5, no.7, pp.12-26, 2013. DOI: 10.5815/ijitcs.2013.07.02 